\title{
The low-frequency sound speed of fluid-like gas-bearing sediments
}

\author{
Preston S. Wilson \\ Applied Research Laboratories, The University of Texas at Austin, P.O.Box 8029, Austin, Texas 78713-8029 \\ and Department of Mechanical Engineering, The University of Texas at Austin, 1 University Station C2200, Austin, \\ Texas 78712-0292 \\ pswilson@mail.utexas.edu \\ Allen H. Reed and Warren T. Wood \\ Marine GeoSciences Division, Naval Research Laboratory, Stennis Space Center, Mississippi 39529 \\ allen.reed@nrlssc.navy.mil,warren.wood@nrlssc.navy.mil \\ Ronald A. Roy \\ Department of Aerospace and Mechanical Engineering, Boston University, Boston, Massachusetts 02215 \\ ronroy@bu.edu
}

\begin{abstract}
The low-frequency sound speed in a fluid-like kaolinite sediment containing air bubbles was measured using an acoustic resonator technique and found to be $114 \mathrm{~m} / \mathrm{s}$ with negligible dispersion between 100 and $400 \mathrm{~Hz}$. The sediment's void fraction and bubble size distribution was determined from volumetric images obtained from x-ray computed tomography scans. A simplified version of Wood's effective medium model, which is dependent only upon the ambient pressure, the void fraction, the sediment's bulk mass density, and the assumption that all the bubbles are smaller than resonance size at the highest frequency of interest, described the measured sound speed.

(C) 2008 Acoustical Society of America

PACS numbers: 43.30.Ma [GD]

Date Received: December 7, 2007

Date Accepted: January 22, 2008
\end{abstract}

\section{Introduction}

The acoustic properties of gassy sediments are important in a diverse range of marine applications including sonar and mine hunting, ${ }^{1}$ civil and petroleum engineering, ${ }^{2}$ and the interpretation of seismic surveys, ${ }^{3}$ among others. A number of studies have been performed regarding scattering from, and sound propagation and attenuation in, gas-bearing sediments. Examples are Refs. 4-7. Despite these and other similar studies, the various models for sound propagation in gassy sediments ${ }^{8-10}$ have gone largely unverified. Wilkens and Richardson ${ }^{11}$ investigated the gas-bearing sediments in Eckernförde Bay and observed resonant bubble acoustic effects consistent with their measured bubble size distribution and the predictions of the Anderson/ Hampton model, ${ }^{8}$ hereafter referred to as AH. Unfortunately, heterogeneous acoustic measurement techniques, and possibly heterogeneous gas distributions prevented an unambiguous characterization of the sediment acoustic properties. Recently, Best et al. ${ }^{6}$ obtained wide band sound speed and attenuation measurements on a gassy sediment and enough environmental characterization data to compare the acoustic measurements to the predictions of $\mathrm{AH}$. They found good agreement between measured and modeled acoustic attenuation (using a best fit bubble size distribution) but found poor agreement between measured and modeled sound speed. Again, heterogeneity of the bubble distribution, and deviation from spherical bubbles were cited.

In the present study, we sought to minimize the experimental complexity and uncertainty, and conducted a laboratory experiment with reconstituted kaolinite sediments that contained entrained air bubbles. Low-frequency (below $1 \mathrm{kHz}$ ) sound speed measurements were obtained with an acoustic resonator technique. A high-frequency $(400 \mathrm{kHz})$, bistatic sound 


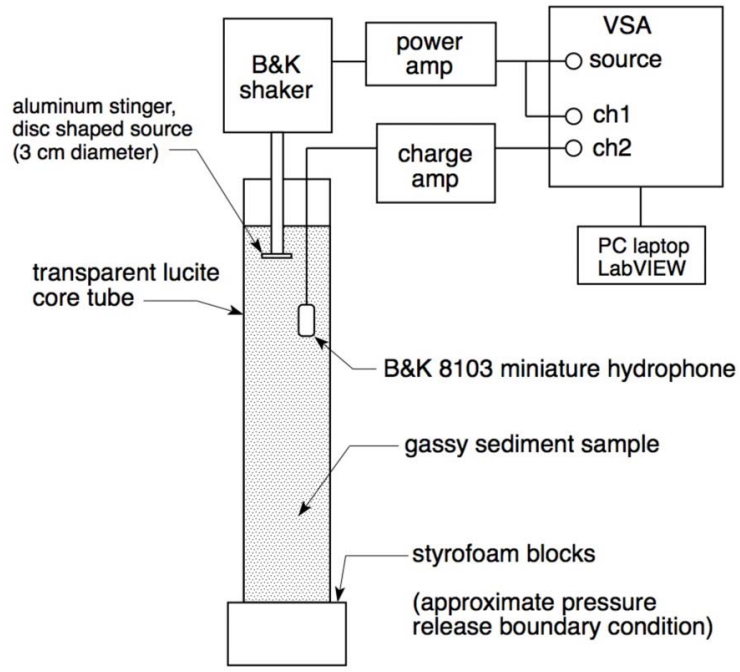

Fig. 1. (Color online) The acoustic resonator and associated apparatus used to obtain the low-frequency sound speed measurements.

speed measurement was attempted but failed due to excess attenuation. High-resolution x-ray computed tomography imagery was used to determine the bubble size distribution and overall void fraction. The fluid-like kaolinite sediment, which had bubbles of primarily spherical and spheroidal shape, exhibited a nondispersive sound speed that was accurately described by a simplified version of Wood's equation. ${ }^{12}$ All measured sound speeds were at frequencies well below the lowest individual bubble resonance frequency (IBRF)

\section{Description of the apparatus and measurement procedure}

The sediment sample was prepared inside an acrylic core tube of circular cross section with $6.1 \mathrm{~cm}$ outer diameter, $1 \mathrm{~mm}$ wall thickness, and $0.463 \mathrm{~m}$ length. A latex rubber membrane (thickness $=0.127 \mathrm{~mm}$ ) was used to close the bottom of the tube. Dried kaolinite was mixed with de-ionized fresh water to a $65 \%$ water mass fraction. Air bubbles were entrained during the sample preparation. A measurement of the high frequency $(400 \mathrm{kHz})$ sound speed was attempted using a pulse transmission technique and apparatus described in Refs. 11, 13, and 14, but it failed due to excess attenuation in the sediment. The low-frequency sound speed was determined using the acoustic resonator shown in Fig. 1. The sample-filled core tube was transferred to the acoustic apparatus and positioned as shown. The air-sediment interface at the top and a styrofoam block at the bottom provided pressure release acoustic boundary conditions to a high degree of approximation, as described in Ref. 15. Acoustic standing waves were generated with a 3-cm-diam aluminum piston that was attached to an electromechanical shaker via an aluminum stinger. Band-limited periodic chirps were produced by a vector signal analyzer (VSA) and directed to the shaker through a power amplifier. The source signal was also digitized by the VSA. The piston was positioned a few centimeters below the upper air-sediment interface. The acoustic pressure inside the sediment was received with a miniature hydrophone, positioned a few centimeters below the source. The received signals were bandpass filtered $(10-10 \mathrm{kHz})$ and amplified with a charge amplifier, and digitized by the VSA. According to manufacturer specifications, the source velocity and receiver pressure responses were flat to within less than $\pm 1 \mathrm{~dB}$ in the experimental frequency range.

Acoustic pressure spectra were then calculated onboard the VSA by way of a transfer function between the received acoustic pressure and the excitation signal using 20 spectral averages. This preserved the pressure magnitude and phase relative to the excitation signal and 
Table 1. Physical properties used in the evaluation of the elastic waveguide model.

\begin{tabular}{lcccc}
\hline \hline $\begin{array}{l}\text { Longitudinal sound } \\
\text { speed }(\mathrm{m} / \mathrm{s})\end{array}$ & $\begin{array}{c}\text { Transverse sound speed } \\
(\mathrm{m} / \mathrm{s})\end{array}$ & $\begin{array}{c}\text { Wall material } \\
\text { density }\left(\mathrm{kg} / \mathrm{m}^{3}\right)\end{array}$ & $\begin{array}{c}\text { Inner wall } \\
\text { radius }(\mathrm{m})\end{array}$ & $\begin{array}{c}\text { Outer wall } \\
\text { radius }(\mathrm{m})\end{array}$ \\
\hline$c_{l}=2664$ & $c_{\mathrm{t}}=1087$ & $\rho_{w}=1185$ & $b=0.0295$ & $d=0.0305$ \\
\hline \hline
\end{tabular}

yielded the coherence function. The number of averages and the amplitude of the excitation were chosen to achieve a near-unity coherence function at all frequencies of interest, which guaranteed a high signal-to-noise ratio and ensured linear bubble behavior. ${ }^{16}$ Peak pressures inside the sediment were typically less than $160 \mathrm{~dB}$ re $1 \mu \mathrm{Pa}$.

Immediately following the acoustic resonator measurements, the sediment sample was transferred to a Universal Systems HD-500 micro-computed x-ray tomography system. ${ }^{17}$ Three-dimensional imaging scans (107 $\mu \mathrm{m}$ voxel size) were obtained over approximately $1 / 3$ of the total sediment volume. A three-dimensional rendering of a portion of the scan is shown in $\mathrm{Mm}$ 1. The sediment contained relatively evenly dispersed gas bubbles of primarily spherical or spheroidal bubbles. Image analysis software was used to calculate total gas volume $V_{\text {gas }}$ and bubble size distribution. Knowledge of the scanned sediment core tube volume $V_{\text {tot }}$ was used to extrapolate global sediment void fraction $\chi=V_{\text {gas }} / V_{\text {tot }}$. The measured void fraction was $\chi$ $=0.0045$ and the void fraction measurement uncertainty was \pm 0.001 due to the voxel size.

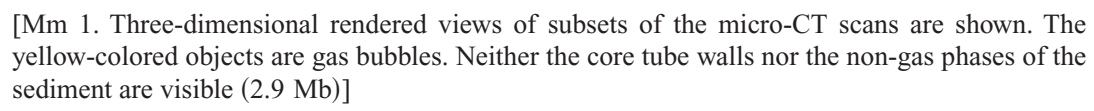

The bulk mass density $\rho_{\text {sed }}$ of gas-free sediment material was measured with a Quantachrome Penta pycnometer. Five samples taken from just below and five from $25 \mathrm{~cm}$ below the air-sediment interface were measured. No significant difference was found between the upper and lower locations. The mean density was $1581.4 \mathrm{~kg} / \mathrm{m}^{3}$ and the range of variation of the five samples was $\pm 5.6 \mathrm{~kg} / \mathrm{m}^{3}$. The density measurement uncertainty was $1 \mathrm{ppt}$ (given in pycnometer's specifications).

\section{Accounting for the elastic waveguide effect}

In a gas-filled acoustic resonator, the walls are effectively rigid. In a liquid-filled resonator, there is significant coupling between the fill-liquid and the tube. The result is a reduced sound speed relative to that observed in an unconfined environment. This effect is hereafter referred to as the elastic waveguide effect. An exact analytic model ${ }^{18}$ for sound propagation in a finitethickness elastic-walled, fluid-filled cylindrical tube was used to relate the speeds observed in the resonator to the intrinsic sound speed the material would exhibit in an unconfined environment. This procedure (and its validity) is discussed in Ref. 15 and summarized here. Equation (A1) of Ref. 15 is the dispersion relation for the resonator waveguide. The intrinsic sound speed $c_{0}$ of the liquid that fills the resonator is an input parameter to Eq. (A1). The phase speed $c_{\mathrm{ph}}$ of the plane wave mode is an output. The resonator measurements described here yield the effective phase speed $c_{\text {eff }}$ observed inside the waveguide. The intrinsic sound speed $c_{0}$ is then varied in Eq. (A1) until the model output $c_{\mathrm{ph}}$ matches the measured value $c_{\text {eff. }}$. The value of $c_{0}$ that achieves the match is reported as the intrinsic sound speed of the sample. The physical parameters used in the elastic waveguide model [Eq. (Al), Ref 15] are given in Table 1. The sound speeds for the tube walls were initially taken from the literature, but the similar values that were ultimately used were obtained via resonator calibration with filtered degassed fresh water.

\section{Model of sound propagation in fluid-like gas-bearing sediment}

The sediment in this experiment was fluid-like. It flowed readily and its shape was not maintained when unsupported. Therefore, Wood's model ${ }^{12}$ for the sound speed in a bubbly liquid was 


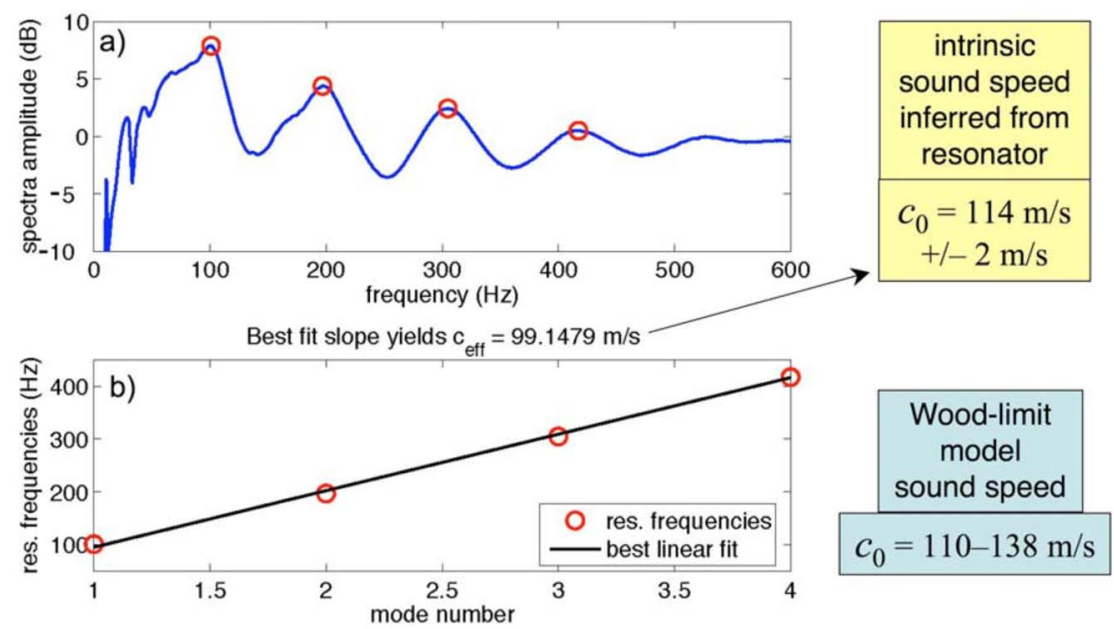

Fig. 2. (Color online) The resonator spectrum obtained for the kaolinite sediment. The four lowest resonance frequencies are identified with circles. (b) The effective sound speed $c_{\text {eff }}$ inside the gassy kaolinite-filled waveguide is inferred from these frequencies, where a least-squares fit yields $c_{\text {eff }}=99.1 \mathrm{~m} / \mathrm{s}$. After correcting for the elastic waveguide effect, the intrinsic sound speed inferred from the measurements was $c_{0}=114 \mathrm{~m} / \mathrm{s} \pm 2 \mathrm{~m} / \mathrm{s}$, where the range is due to finite spectral resolution and the uncertainty in the resonator length $L$. The Wood limit model [Eq. (1)] predicted $c_{0}=110-138 \mathrm{~ms}$, where the range is due to the uncertainty of the void fraction.

used, which requires that the excitation frequency be well below the resonance frequency of the largest bubble. For $0.002<\chi<0.94$, and isothermal conditions (a good approximation for these bubble sizes) the model reduces to ${ }^{19}$

$$
c_{\text {lfgs }}=\sqrt{\frac{P_{\mathrm{atm}}+\rho_{\mathrm{sed}} g(L / 2)}{\chi(1-\chi) \rho_{\mathrm{sed}}}},
$$

where $c_{\text {lfgs }}$ is the low-frequency sound speed in the gassy sediment, $P_{\text {atm }}=101.3 \mathrm{kPa}$ is the atmospheric pressure, $\rho_{\text {sed }}$ is the sediment mass density, $g=9.81 \mathrm{~m} / \mathrm{s}^{2}$ is the acceleration of gravity, $L$ is the length of the resonator, and $\chi$ is the void fraction. The terms in the numerator account for the mean dependence of the bubble compressibility on the local hydrostatic pressure. In this experiment, the largest bubble radius observed was $4.6 \mathrm{~mm}$. The corresponding bubble resonance frequency is $4.91 \mathrm{kHz}$, determined by Eq. (1) of Ref. 8 using a conservatively low value for sediment shear modulus $\left(G=10^{4} \mathrm{~Pa}\right)$, also from Ref. 8. The highest frequency in these experiments was $0.8 \mathrm{kHz}$, which rendered the low-frequency approximation valid. Note that even if $G$ was set to zero, the lowest bubble resonance frequency would still be about six times higher than the highest acoustic excitation frequency. The simplified form of Eq. (1) is used because it best illustrates the physical nature of sound propagation in near-surface gas-bearing fluid-like sediments.

\section{Results}

The measured resonator spectrum is shown in Fig. 2(a), where four resonance frequencies are identified. The effective sound speed $c_{\text {eff }}$ is inferred from the slope of the curve in Fig. 2(b). The $n$th resonance frequency is given by $f_{n}=\left(c_{\text {eff }} / 2 L\right) n$ and $L$ is the length of the resonator. After correction for the elastic waveguide effect (Sec. 3), the intrinsic gassy kaolinite sound speed was $c_{0}=114 \mathrm{~m} / \mathrm{s} \pm 2 \mathrm{~m} / \mathrm{s}$. The measurement uncertainty was due to the finite spectral resolution and the uncertainty of $L( \pm 1.2 \mathrm{~mm})$. For the length, void fraction and density reported in 
Table 2. Measured and predicted sound speeds.

\begin{tabular}{lcccc}
\hline \hline Sediment type & $\begin{array}{c}\text { Frequency range of } \\
\text { resonator } \\
\text { measurements }(\mathrm{Hz})\end{array}$ & $\begin{array}{c}\text { Low-frequency } \\
\text { resonator sound } \\
\text { speed }(\mathrm{m} / \mathrm{s})\end{array}$ & $\begin{array}{c}\text { Sound speed predicted by } \\
\text { Eq. }(1)(\mathrm{m} / \mathrm{s})\end{array}$ & $\begin{array}{c}400 \mathrm{kHz} \text { pulse } \\
\text { transmission sound } \\
\text { speed }(\mathrm{m} / \mathrm{s})\end{array}$ \\
\hline Gassy kaolinite & $100-550$ & $114 \pm 2$ & $110-138$ & $\mathrm{n} / \mathrm{a}$ due to excess \\
attenuation \\
$\begin{array}{l}\text { Degassed filtered water } \\
\text { (calibration) }\end{array}$
\end{tabular}

Sec. 2, the model [Eq. (1)] predicts a low-frequency gassy sediment sound speed between 110 and $138 \mathrm{~m} / \mathrm{s}$. The range of the prediction is due to the void fraction measurement uncertainty. Excellent agreement is observed between the measurement and the model. A summary of the measured and predicted sound speeds appear in Table 2.

\section{Conclusions}

The two major conclusions of this work are: (1) Resonator measurements of gassy sediments yield the sub-IBRF sound speed even where high-frequency pulse transmission measurements fail. (2) A simplified version of Wood's equation describes the sound speed in shallow gasbearing fluid-like sediments in which the IBRF of the largest bubble is well above the frequency of the acoustic excitation, and the bubbles are evenly distributed throughout the sample. The gas phase dominates the compressibility. The sound speed is dependent only on the void fraction, the sediment mass density, and the local hydrostatic pressure. It does not depend at all on the material properties of the sediment grains and the fluid, other than through the bulk sediment density. This will cease to be true as the local hydrostatic pressure increases, which in turn decreases the compliance contrast between the gas phase and the longitudinal and shear compliances of the gas-free phase of the sediment. The range of applicability of this simple model is the subject of current work.

\section{Acknowledgments}

This work was supported by the University of Texas at Austin Cockrell School of Engineering, the Naval Research Laboratory-Stennis Space Center, Program Element No. 0601153N, and the Office of Naval Research Coastal Geosciences and Ocean Acoustics programs.

\section{References and links}

${ }^{1}$ D. R. Jackson and M. D. Richardson, High-Frequency Seafloor Acoustics (Springer, New York, 2007).

${ }^{2}$ A. M. Davis, "Shallow gas: An overview," Cont. Shelf Res. 12, 1077-1079 (1992).

${ }^{3}$ S. N. Domenico, "Effect of brine-gas mixture on velocity in an unconsolidated sand reservoir," Geophysics 41, 882-894 (1976).

${ }^{4}$ F. A. Boyle and N. P. Chotiros, "A model for high-frequency acoustic backscatter from gas bubbles in sandy sediments at shallow grazing angles," J. Acoust. Soc. Am. 98, 531-541 (1995).

${ }^{5}$ A. P. Lyons, M. E. Duncan, A. L. Anderson, and J. A. Hawkins, "Predictions of the acoustic scattering response of free-methane bubbles in muddy sediments," J. Acoust. Soc. Am. 99, 163-172 (1996).

${ }^{6}$ A. I. Best, M. D. J. Tuffin, J. K. Dix, and J. M. Bull, "Tidal height and frequency dependence of acoustic velocity and attenuation in shallow gassy marine sediments," J. Geophys. Res. 109, B08101 (2004).

${ }^{7}$ T. Gardner, "Modeling signal loss in surficial marine sediments containing occluded gas," J. Acoust. Soc. Am. 113, 1368-1378 (2003).

${ }^{8}$ A. L. Anderson and L. D. Hampton, “Acoustics of gas-bearing sedimets. II. Measurements and models," J. Acoust. Soc. Am. 67, 1890-1903 (1980).

${ }^{9}$ A. Bedford and M. Stern, “A model for wave propagation in gassy sediments,” J. Acoust. Soc. Am. 73, 409-417 (1983).

${ }^{10}$ D. M. J. Smeulders and M. E. H. Van Dongen, "Wave propagation in porous media containing a dilute gasliquid mixture: Theory and experiments," J. Fluid Mech. 343, 351-373 (1997).

${ }^{11}$ R. H. Wilkens and M. D. Richardson, "The influence of gas bubbles on sediment acoustic properties: In situ, laboratory, and theoretical results from Eckernforde Bay, Baltic sea," Cont. Shelf Res. 18, 1859-1892 (1998).

${ }^{12}$ A. B. Wood, A Textbook of Sound, 1st ed. (MacMillan, New York, 1930). 
${ }^{13}$ M. D. Richardson and K. B. Briggs, "In situ and laboratory geoacoustic measurements in soft mud and hardpacked sand sediments: Implications for high-frequency acoustic propagation and scattering," Geo-Mar. Lett. 16, 196-203 (1996).

${ }^{14}$ K. L. Williams, D. R. Jackson, E. I. Thorsos, D. Tang, and S. G. Schock, "Comparison of sound speed and attenuation measured in a sandy sediment to predictions based on the Biot theory of porous media," IEEE J. Ocean. Eng. 27, 413-428 (2002).

${ }^{15}$ P. S. Wilson, A. H. Reed, J. C. Wilbur, and R. A. Roy, "Evidence of dispersion in an artificial water-saturated sand sediment," J. Acoust. Soc. Am. 121, 824-832 (2007).

${ }^{16}$ J. S. Bendat and A. G. Piersol, Engineering Applications of Correlation and Spectral Analysis, 2nd ed. (Wiley, New York, 1993).

${ }^{17}$ http://www7430.nrlssc.navy.mil/facilities/CTScanner/hd500mct.htm (last viewed November 2007).

${ }^{18}$ V. A. Del Grosso, "Analysis of multimode acoustic propagation in liquid cylinders with realistic boundary conditions-Application to sound speed and absorption measurements," Acustica 24, 299-311 (1971).

${ }^{19} \mathrm{~L}$. van Wijngaarden, "On the equations of motion for mixtures of liquid and gas bubbles," J. Fluid Mech. 33, 465-474 (1968). 